mer weitgehend verschont geblieben von den tragischen Folgen seiner Expansion über die Ozeane. Die anderen hatten in Form von Krankheiten und Seuchen, von Ausbeutung und ökologischen Zerstörungen die Bürden zu tragen. Nun aber scheint sich der Trend zu wenden. Zum ersten Male sehen sich jetzt auch die Länder des Nordens mit den bitteren Folgen der Verwestlichung der Welt konfrontiert. Einwanderungs- und Bevölkerungsdruck sowie ein um sich greifendes Denken in Stammesbegriffen, vor allem aber die Folgen der weltweiten Industrialisierung für die Umwelt drohen die Lebensweise des Nordens zu destabilisieren. Es sieht so aus, als schließe sich der Kreis, der sich mit der Ausfahrt des Kolumbus geöffnet hatte, am Ende dieses Jahrhunderts wieder. Daher entwirft der Norden Wege und Möglichkeiten des Selbstschutzes und des weltweiten Risiko-Managements. Die rationale Planung des Planeten wird zu einer Angelegenheit der Sicherheit des Nordens.

Die so hochgelobte Herrschaft des (westlichen) Menschen über die Natur läßt ja allerhand zu wünschen übrig. Wissenschaft und Technologie verändern erfolgreich in weitem Umfang die Natur, aber bislang mit sehr unerwünschten und unvorhersehbaren Folgen. Nur wenn diese Folgen unter Kontrolle gebracht wären, ließe sich mit einiger Berrechtigung behaupten, der Mensch beherrsche die Natur. An diesem Punkt setzen die technokratischen Umweltschützer an. Von daher ist globales Umweltmanagement nichts weniger als Kontrolle zweiter Ordnung: Es muß sich ein höheres Niveau der Beobachtung und des Eingriffs entwickeln, um die Konsequenzen der Kontrolle über die Natur zu kontrollieren. Solch ein Schritt wird umso zwingender, je länger der Trend, die Welt zu einer engen,' in sich verwobenen und expandierenden

\title{
Nachhaltigkeit - eine neue Kompromißformel?
}

Je nachdem wie der Begriff der >Nachhaltigen Entwicklung < definiert und interpretiert wird, ergeben sich unterschiedliche Politikansätze und Handlungsnotwendigkeiten. Auch wenn es generell zu begrïßen ist, daß sich die Enquete-Kommission »Schutz des Menschen und der Umwelt « des deutschen Bundestages zu diesem Leitbild bekennt, so sollte die vorgenommene Präzisierung des Begriffs zu denken geben. Ich möchte dies zum Anlaß nehmen, auf einige alte und neue Klippen hinzuweisen, die im Spannungsfeld von Nachhaltigkeit und wirtschaftlicher Entwicklung beachtet werden sollten.

\section{Grundregeln für den Umgang mit Stoffen}

Aus Sicht der Enquete-Kommission ergeben sich vier Grundregeln für den Umgang mit Stoffen bzw. mit Stoffströmen:

»(1) Die Abbaurate erneuerbarer Ressourcen soll deren Regenerationsrate nicht überschreiten (= Aufrechterhaltung der ökologischen Leistungsfähigkeit).

(2) Stoffeinträge in die Umwelt müssen sich an der Belastbarkeit der als Senken dienenden Umweltmedien in allen ihren Funktionen orientieren.

(3) Nicht erneuerbare Ressourcen sollen nur in dem Umfang genutzt werden, in dem ein physisch und funktionell gleichwertiger Ersatz in Form erneuerbarer Ressourcen oder höherer Produktivität der erneuerbaren sowie der nicht erneuerbaren Ressourcen geschaffen wird (= Druck auf ökologisch orientierte Innovationen).

(4) Das Zeitmaß anthropogener Einträge bzw. Eingriffe in die Umwelt muß in einem ausgewogenen Verhältnis zu der Zeit stehen, die die Umwelt zur Reaktion benötigt« (1).

\section{Nachhaltige Ressourcennutzung anstatt Naturerhalt?}

Nachhaltigkeit wird auch in dieser Definition verstanden als Erhalt eines bestimmten, durch den Menschen zu nutzenden, natürlichen Systems (Ressource, Senke) und ist damit streng. anthropozentrisch ausgerichtet. Dies hat weitgehende Konsequenzen für die konzeptionelle Orientierung und Operationalisierung:

- Nachhaltige zukunftsfähige Entwicklung bezieht sich immer nur auf den zu nutzenden Anteil der Natur. Über das Verhältnis von Nutzung und Nichtnutzung (z. B. Naturschutz) werden keine Aussagen getroffen. Dies ist jedoch vor dem Hintergrund der bereits eingetretenen Schädigungen und Funktionsstörungen der natürlichen Systeme ein unverzichtbarer Beitrag zu deren Erhalt.

- Nachhaltige Entwicklung favorisiert naheliegend den Systemerhalt unmittelbar >nützlicher< oder zu nutzender Öko-Systeme (z. B. Wälder, Fischbestände, Wasservorkommen, Ressourcen). Dies setzt eine klare Vorstellung über die Systembeziehungen und Systemgrenzen voraus, um zu eindeutigen Aussagen über die vertretbaren Input-Output Größen zu kommen. Die Komplexität ökosystemarer Zusammenhänge ist jedoch in vielen Fällen durch eine lineare Input-Output Beziehung nicht zu erfassen.

- Mit der generellen Nutzenorientierung der nachhaltigen Wirtschaftskonzepte ist eine implizite Hierarchisierung ökologischer Systemfunktionen verbunden, da diese immer durch die Brille der Produktionsfunktion ge-
Gemeinschaft zu machen, unvermindert fortbesteht. Da die ungebrochene Kraft der Entwicklungsideologie eine Verlangsamung der weltweiten Industrialisierungsdynamik undenkbar erscheinen läßt, wird es unausweichlich, die Transformation der Natur in globalem Maßstab zu steuern. In diesem Licht konnte die Zeitschrift Scientific American die folgenden Fragen zu Schlüsselfragen künftiger Entscheidungen erheben: »Zentrale Fragen harren der Antwort: welche Art von Planet wünschen wir? Welche Art von Planet können wir bekommen?... Auf welcher Höhe sollte die Artenvielfalt aufrechterhalten werden? Sollte die Größe oder die Wachstumsrate der Menschheit verringert werden ...? In welchem Umfang ist Klimaveränderung akzeptabel? «Wenn es schon keine Grenzen des Wachstums mehr geben soll, dann auch gewiß keine des Hochmuts.

Wolfgang Sachs, Wuppertal

sehen werden. Das Hauptaugenmerk liegt auf Umweltverbräuchen und deren möglicher Reduzierung (2),

Trotz dieser einschränkenden Bemerkungen können aus dem Nachhaltigkeitsparadigma Orientierungen abgeleitet werden. Wichtig erscheint mir hierbei die auch von der EnqueteKommission vorgenommene Unterscheidung zwischen erneuerbaren und nicht-erneuerbaren Ressourcen.

Orientierung an der Regenerationsrate und der Regenerationsfähigkeit

Für erneuerbare Ressourcen und das Problem der Stoffeinträge werden in der Grundtendenz ähnliche Aussagen getroffen wie sie bereits von Daly (3) gemacht wurden, allerdings etwas präziser formuliert wurden:

(1) Die Ernte-Raten dürfen die Regenerationsrate des genutzten Öko-Systems nicht überschreiten.

(2) Die Einträge durch Abfall/Emissionen dürfen die Assimilationsfähigkeit des Systems nicht überschreiten und seine Regenerationsfähigkeit nicht einschränken.

Durch diese Definitionen zieht sich der Grundgedanke, daß der Verbrauch von erneuerbaren Ressourcen immer ins Verhältnis zur natürlichen Aufnahmekapazität zu setzen ist. Diese ist jedoch keine absolute Grenze, sondern kann durch die Stärkung der Regenerationspotentiale und durch geschicktes Einklinken in natürliche Stoffkreisläufe erweitert werden. Je mehr nicht-regenerative Stoffe durch regenerative Stoffe ersetzt werden, desto stärker können die anthropogenen Stoffkreisläufe an die natürlichen Regenerationsraten >angebunden< werden. So verändert die Nutzung pflanzlicher Stoffe die $\mathrm{CO}_{2}$-Bilanz der Atmosphäre nicht negativ. Bei ihrer Zersetzung oder Verbrennung wird nur so viel $\mathrm{CO}_{2}$ frei, wie die Pflanzen vorher gebunden haben. 
Diese öko-systemischen Aspekte der Regenerierbarkeit wurden in den bisherigen Ansätzen zum Stoffstrommanagement weitgehend vernachlässigt. Sie hätte auch weitgehende Konsequenzen für die zukünftige Zusammensetzung der Enquete. Eine veränderte, nämlich weitgehend bio-organische Stoffbasis der industriellen Produktion und ein anderer Umgang mit diesen Stoffen (z. B. die Rolle sanfter Biotechniken) würde auch implizieren, die Bauern und die Landwirtschaft als stoffpolitischen Akteur (wieder) wahrzunehmen.

\section{Produktivität als Maßstab?}

Für die Nutzung der nicht-erneuerbaren Ressourcen wird in der Definition der rein stoffpolitische Maßstab verlassen und auf einen »funktionell gleichwertigen Ersatz ... höherer Produktivität « verwiesen. Angesichts des tatsächlichen Konfliktes, der sich hinter dieser Formulierung verbirgt, stellt dies zunächst eine semantische Spitzenleistung dar. Trotzdem ist dies ein schlechter Formelkompromiß, den ich für die weitere Arbeit der Enquete nicht für tragfähig halte.

Zunächst wird mit einer solchen Formulierung die ökologische Problemdimension ausgeblendet. Die Nutzung fossiler Brennstoffe ist eine Basis der industriellen Entwicklung. Hier nicht nur zu relativen sondern zu absoluten Reduzierungen der Stoffumsätze zu kommen, ist ein Kernbestandteil des ökologischen Anliegens. Die ökosystemare Belastung im globalen $\mathrm{Maßstab}\left(\mathrm{CO}_{2}\right.$-Problematik) hängt z. B. ursächlich mit dem Raubbau der Industriegesellschaft an den sunterirdischen Wäldern (Öl, Kohle, Erdgas) zusammen. Wenn nicht innerhalb von 200 Jahren das > verpulvert $<$ würde, was die $\mathrm{Na}$ tur in Jahrmillionen aufgebaut hat, so hätten wir nicht mit derartigen Ungleichgewichten in den Naturhaushalten zu tun. Hier wieder ins Gleichgewicht zu kommen (auch was das Zeit$\mathrm{ma} ß$ der anthropogenen Einträge betrifft), ist ein zentrales Ziel der nachhaltigen Entwicklungskonzepte.

Dieser Problemhintergrund wird von einigen Mitgliedern der Enquete-Kommission nicht anerkannt. Stattdessen wird unterstellt, daß die Minimierung des Stoffeinsatzes um ihrer selbst willen zum umweltpolitischen Ziel erhoben wird (4). Und an gleicher Stelle wird das Schreckgespenst eines > Umweltschutzes um jeden Preis< aus der politischen Asservatenkammer geholt: »Ein solches Vorgehen würde Kriterien wie Sozial- oder Ökonomieverträglichkeit ausblenden, $\mathrm{zu}$ einer effizienzmindernden schleichenden Transformation des marktwirtschaftlichen Systems führen, eine Exportwirtschaft, die dies im Alleingang versucht, mit großer Wahrscheinlichkeit vom Welthandel abkoppeln und liefe damit Gefahr, wichtigen Nachhaltigkeitsanforderungen $\mathrm{zu}$ widersprechen « (5). Mit diesen Ausführungen wird deutlich, warum die stoffpolitischen Reduktionsziele an die Produktivitätsfortschritte angebunden werden sollen, wie dies auch in Punkt 3 der oben vorgestellten Enquete-Definition befürwortet wird. Von der Logik der Argumentation her ist dies nicht weniger als die Forderung an die Natur, sie solle sich gefälligst dem ökonomischen Leistungsvermögen anpassen und nicht umgekehrt. Ein Paradigmenwechsel - der die Anpassungserfordernisse von den Grenzen und der Tragekapazität der natürlichen Potentiale her denkt - ist damit ausgeschlossen. Insofern sehe ich auch in den Definitionsbemühungen der Enquete in Sachen Nachhaltigkeit keinen wesentlichen Fortschritt.

Für das wichtige Handlungsfeld der nicht-erneubaren Ressourcen sollte ohne Einschränkung der Grundsatz gelten: Nichterneuerbare Ressourcen sollten in dem Maße abgebaut werden, wie es möglich ist, ein erneuerbares Ausgleichspotential aufzubauen.

Mit dieser Option ist auch das grundlegende Verhältnis von erneuerbaren und nicht-erneuerbaren Ressourcen angesprochen, welches sicherlich nicht nur projektbezogen oder an einzelnen Stoffgruppen bestimmt werden kann sondern auch auf ökosystemischer Ebene zu klären wäre, da erneuerbare Ressourcen über ihre unmittelbare, stoffliche Verwendbarkeit auch positive und negative Rückkopplungen zur Bio- und Atmosphäre haben. Diese Option muß für die Wirtschaft nicht nur einschränkenden Charakter haben. Hierin liegen auch neue Entwicklungsmöglichkeiten, die ich abschließend kurz umreißen will.

\section{Nachhaltigkeit}

und Innovationsorientierung

Das Gegenmodell zu einer Raubbau-Ökonomie ist eine Steady-State-Ökonomie, verstanden als ein Wirtschaften, daß sich im wesentlichen an der Leistungsfähigkeit und Regenerationsfähigkeit der Natur orientiert (6). Die Gleichgewichtsvorstellung eines derartigen Ansatzes sollte nicht stationär sondern evolutionär sein, $d$. h. daß die Natur nicht nur als Grenze sondern auch als produktiver, noch zu entwickelnder Faktor gesehen wird. Mit einer solchen Sichweise ist auch eine gewisse Anschlußfähigkeit an dynamische, innovative Entwicklungen des Wirtschaftsprozesses gegeben. Allerdings brauchen wir einen neuen Innovationstypus. Die Erfahrungen mit der Rationalisierung der Arbeit, die in ihrem Extrem dazu geführt hat, daß die Arbeit in hunderte Bestandteile zerlegt, neu zusammengesetzt und zeitlich verdichtet wurde, sollte eigentlich Mahnung genug sein, um nicht einfach einem neuem Rationalisierungsprogramm für die Natur das Wort zu reden. Insofern sehe ich einigen Diskussionsbedarf hinsichtlich der Frage, welche Effizienztechniken zum Einsatz kommen sollen. Unisono wird derzeit von ökologisch bewußten Menschen der 1 Liter Sparmotor eingefordert, ohne sich überhaupt die Frage zu stellen, mit welchen Materialien diese Leistungsverdichtung erreicht werden kann.
Es muß eine generelle Blickwende in der Technologiepolitik eingeleitet werden. Sustainibility erfordert gerade die Entwicklung völlig neuer Recycling- und Allianztechniken, die die natürliche Produktivität der Ökosysteme nicht nur nutzt, sondern sie sogar noch fördert. Mit einer derartigen Strategie (Effizienz - Erhalt - Allianz) könnte auch ein Teil des ungeheuren Innovationsdrucks genommen werden, der derzeit auf den Effizienzstrategien-lastet und meiner Meinung nach auch zu gesellschaftlichen Handlungsblockaden führt. In wissenschaftlicher Hinsicht macht ein derartiger StrategieMix eine stärkere Hinwendung zu den erfahrungsorientierten Wissenschaften notwendig. Auch die Innovationsorientierung müßte weiter gefächert werden. Zukünftige Technikentwicklung darf sich bei ihrer ökologischen Orientierung nicht alleine auf Effizienz konzentrieren, sondern muß auch Kriterien wie Anpassungsfähigkeit, Synergiefähigkeit und Erhalt des Ordnungsniveaus berücksichtigen (7)

Rainer Lucas, Wuppertal

\section{Anmerkungen}

(1) Enquete-Kommission »Schutz des Menschen und der Umwelt « des Deutschen Bundestages: Die Industriegesellschaft gestalten. Perspektiven für den nachhaltigen Umgang mit Stoff- und Materialströmen. S. 32

(2) vgl. hierzu auch die Selbstdarstellung des Projektes >Zukunftsfähiges Deutschland am Wuppertal - Institut, Wuppertal 1994

(3) Daly H.E: 1993: Sustainable Growth: An impossibility Theorem. In: Daly, H.E., Townsend, N. (Ed.) Valuing the Earth. London, S. 271

(4) Klemmer, P.: Ressourcen- und Umweltschutz um jeden Preis?, in: Voss, G. (Hg.): Sustainable Developement - Leitziel auf dem Weg in das 21. Jahrhundert. Köln 1994, 49

\section{(5) ebd. S. 49}

(6) siehe Daly, H.E. 1993: Introduction to Essays toward a Steady-State-Economy. In: Daly, H.E., Townsend, N. a.a. O., $11-47$

(7) vgl. hierzu ausführlich: v. Gleich, Arnim: Leitbilder einer Stoffpolitik, IÖW - Diskussionspapier 23/94

\section{Bitte beachten Sie den Redaktionsschluß des nächsten Informationsdienstes:}

\section{Februar 1995}


(c) 20I0 Authors; licensee IÖW and oekom verlag. This is an article distributed under the terms of the Creative Commons Attribution Non-Commercial No Derivates License (http://creativecommons.org/licenses/by-nc-nd/3.o/), which permits unrestricted use, distribution, and reproduction in any medium, provided the original work is properly cited. 